\title{
A Study of Universal Thermodynamics in Brane World Scenario
}

\author{
Saugata Mitra, Subhajit Saha, and Subenoy Chakraborty \\ Department of Mathematics, Jadavpur University, Kolkata, West Bengal 700032, India \\ Correspondence should be addressed to Saugata Mitra; saugatamitra20@gmail.com
}

Received 16 October 2014; Revised 9 December 2014; Accepted 9 December 2014

Academic Editor: Kingman Cheung

Copyright (C) 2015 Saugata Mitra et al. This is an open access article distributed under the Creative Commons Attribution License, which permits unrestricted use, distribution, and reproduction in any medium, provided the original work is properly cited. The publication of this article was funded by SCOAP ${ }^{3}$.

A study of Universal thermodynamics is done in the framework of RSII brane model and DGP brane scenario. The Universe is chosen as FRW model bounded by apparent or event horizon. Assuming extended Hawking temperature on the horizon, the unified first law is examined for perfect fluid (with constant equation of state) and Modified Chaplygin Gas model. As a result there is a modification of Bekenstein entropy on the horizons. Further the validity of the generalized second law of thermodynamics and thermodynamical equilibrium are also investigated.

From astrophysical observations [1-5], it is now well established that our Universe is going through an accelerating phase. It is speculated that this cosmic acceleration is driven by some invisible fluid (known as dark energy (DE)) having strong repulsive gravitational effect and has come into action only in recent past. But till now the nature of DE is completely unknown to us and is an unresolved problem in modern theoretical physics [6-12]. On the other hand people have tried to modify Einstein's gravity theory itself as an alternative way of resolving this problem. Brane world scenario is one of such models related to gravity theory in higher dimensions. In RSII brane model [13-15], our Universe is a positive tension 3-brane embedded in a 5-dimensional AdS bulk space-time. The standard model fields are confined on the brane while gravity can propagate in the bulk also. So the effective gravity on the brane is different from the standard Einstein gravity due to the existence of extra dimension. Another simple and well studied model of brane gravity is the Dvali-GabadadzePorrati (DGP) brane world model [16-19]. In contrast to RSII model where the extra dimension is of finite size, in DGP brane model our 4-dimensional world (3-brane) is embedded in a space-time with an infinite size extra dimension with the motivation of resolving the cosmological constant problem as well as problems in supersymmetry breaking [16-19]. Usually in this model, FRW brane is embedded in a 5-dimensional Minkowski bulk.
Black hole thermodynamics [20-22] and AdS/CFT correspondence [23] established a deep connection between gravity and thermodynamics. Jacobson [24] and Padmanabhan et al. [25-27] also showed the connection between gravity and thermodynamics. Jacobson deduced Einstein's field equations from the Clausius relation for local Rindler horizon. Padmanabhan, on the other hand, showed that the field equations in Einstein gravity for a spherically symmetric space-time can be expressed as the first law of thermodynamics.

From thermodynamical viewpoint, the study of dynamical black hole was initiated by Hayward et al. [28-31]. They introduced the notion of trapping horizon in 4D Einstein gravity for nonstationary spherically symmetric space-times and showed that Einstein's equations are equivalent to the unified first law. Then projecting the unified first law along any tangential direction $(\xi)$ to the trapping horizon, one is able to derive the first law of thermodynamics [32-34] or equivalently Clausius relation of the dynamical black hole. The homogeneous and isotropic FRW Universe may be considered as dynamical spherically symmetric space-time from cosmological viewpoint.

Further, our Universe is considered as a nonstationary gravitational system from the perspective of Universal thermodynamics. The inner trapping horizon coincides with the apparent horizon and one can study Universal thermodynamics using the unified first law. Starting with the unified 
first law, the Friedmann equations with arbitrary spatial curvature were derived by Cai and Kim [35]. They have considered $T=1 / 2 \pi R_{A}$ as the Hawking temperature and $S=\pi R_{A}^{2} / G$ as the Bekenstein entropy on the apparent horizon having radius $R_{A}$. Also using the entropy formulae (not the Bekenstein one) for the static spherically symmetric black hole horizons in Gauss-Bonnet gravity and in more general Lovelock gravity they were also able to obtain the Friedmann equations in those gravity theories. Subsequently, Cai and Cao [32] have shown that Clausius relation does not hold at the apparent horizon of the FRW Universe in scalar-tensor gravity and they concluded that it corresponds to a system of nonequilibrium thermodynamics similar to $f(R)$-gravity (Eling et al. [36]). Cai and Cao [34] also studied thermodynamics of apparent horizon in RSII brane scenario and have obtained non-Bekenstein entropy on the horizon from the Clausius relation and it reduces to Bekenstein entropy in the limit of large horizon radius.

In the present work, we study Universal thermodynamics in both RSII brane model and DGP brane scenario using extended Hawking temperature on the horizon (apparent/ event) and investigate whether the entropy on the horizon is Bekenstein or not for the validity of the unified first law of thermodynamics. Finally, validity of the generalized second law of thermodynamics (GSLT) and thermodynamical equilibrium (TE) is also examined.

We start with homogeneous and isotropic FRW metric as

$$
\begin{aligned}
d s^{2} & =-d t^{2}+\frac{a^{2}(t)}{1-k r^{2}} d r^{2}+R^{2} d \Omega_{2}^{2} \\
& =h_{a b} d x^{a} d x^{b}+R^{2} d \Omega_{2}^{2},
\end{aligned}
$$

where $R=a r$ is the area radius, $h_{a b}=\operatorname{diag}\left(-1, a^{2} /\left(1-k r^{2}\right)\right)$ is the metric of 2 -space $\left(x^{0}=t, x^{1}=r\right)$, and $k=0, \pm 1$ denotes the curvature scalar.

The surface gravity [37],

$$
\kappa=\frac{1}{2 \sqrt{-h}} \partial_{a}\left(\sqrt{-h} h^{a b} \partial_{b} R\right),
$$

for any horizon (with area radius $R_{h}$ ) in FRW model, can be written as

$$
\kappa=-\left(\frac{R_{h}}{R_{A}}\right)^{2}\left(\frac{1-\dot{R}_{A} / 2 H R_{A}}{R_{h}}\right) .
$$

That is,

$$
\begin{aligned}
& \kappa_{h}=-\left(\frac{R_{h}}{R_{A}^{2}}\right)(1-\epsilon), \text { for any horizon, } \\
& \kappa_{A}=-\frac{(1-\epsilon)}{R_{A}}, \text { for apparent horizon, }
\end{aligned}
$$

with $\epsilon=\dot{R}_{A} / 2 H R_{A}$.

Using this form of surface gravity the extended Hawking temperature is defined as [38]

$$
T_{\mathrm{EH}}^{h}=\frac{\left|\kappa_{h}\right|}{2 \pi} .
$$

According to Hayward [28-30] the unified first law can be expressed as

$$
d E=A \psi+W d V
$$

where $E=(R / 2 G)\left(1-h^{a b} \partial_{a} R \partial_{b} R\right)$ is the total energy inside a sphere of radius $R$ and is termed Misner-Sharp energy [28$30,35,39]$. Also the energy flux $\psi$ is termed energy supply vector and $W$ is the work function and they are defined as

$$
\begin{gathered}
\psi_{a}=T_{a}^{b} \partial_{b} r+W \partial_{a} r, \\
W=-\frac{1}{2} \operatorname{trace} T
\end{gathered}
$$

where $T_{a b}$ is the energy momentum tensor.

Further to have a complete thermodynamical study one has to examine the validity of the generalized second law of thermodynamics (GSLT) and thermodynamical equilibrium (TE) on the horizons. For their validity we must have the following inequalities $[40,41]$ :

$$
\begin{gathered}
\frac{\partial}{\partial t} S_{\mathrm{TH}} \geq 0 \quad(\text { for GSLT) } \\
\frac{\partial^{2}}{\partial t^{2}} S_{\mathrm{TH}}<0 \quad(\text { for } \mathrm{TE})
\end{gathered}
$$

where $S_{\mathrm{TH}}=S_{h}+S_{f h}$, with $S_{h}$ and $S_{f h}$ as the horizon entropy and the entropy of the fluid bounded by the horizon, respectively. To obtain fluid entropy $S_{f h}$ one uses Gibb's relation [42-48]:

$$
T_{f} d S_{f h}=d E_{f}+p d V_{h}
$$

where $E_{f}=\left(\rho V_{h}\right)$ is the energy flow across the horizon, $V_{h}=(4 / 3) \pi R_{h}^{3}$ is the volume of the fluid, $(\rho, p)$ are the energy density and thermodynamic pressure of the fluid, and $T_{f}$ is the temperature of the fluid which is assumed to be the same as the extended Hawking temperature on the horizon. As a result the time variation of the fluid entropy is given by

$$
\dot{S}_{f h}=\frac{4 \pi R_{h}^{2}}{T_{\mathrm{EH}}^{h}}(\rho+p)\left\{\dot{R}_{h}-H R_{h}\right\} .
$$

It should be noted that in deriving the above relation we have used the energy conservation relation for the fluid; that is,

$$
\dot{\rho}+3 H(\rho+p)=0 .
$$

In the rest of the paper we will work with units where $8 \pi=$ $1=G$.

RSII Brane World. In a flat, homogeneous, and isotropic FRW brane extended in 5D AdS bulk, the equivalent Friedmann equations (without dark radiation term) are given by [34, 49]

$$
\begin{aligned}
H^{2} & =\frac{\rho_{t}}{3}, \\
\dot{H} & =-\frac{1}{2}\left(\rho_{t}+p_{t}\right),
\end{aligned}
$$


where $\rho_{t}=\rho+\rho_{e}, p_{t}=p+p_{e}$, and the effective energy density $\rho_{e}$ and the effective pressure $p_{e}$, due to embedding of the brane to the bulk, have the expressions

$$
\begin{gathered}
\rho_{e}=\frac{\kappa_{5}^{4} \rho^{2}}{12}, \\
\rho_{e}+p_{e}=\frac{\kappa_{5}^{4} \rho(\rho+p)}{9},
\end{gathered}
$$

so that we have

$$
\frac{\partial}{\partial t}\left(\rho_{e}+p_{e}\right)=\frac{\kappa_{5}^{4} \rho^{2}}{9}\left(f_{A}-4 H v_{A}\right),
$$

where $\kappa_{5}$ is 5 -dimensional gravitational coupling constant and is related to brane tension $(\lambda)$ and 4-dimensional gravitational coupling constant by the relation $\kappa_{5}^{4}=\kappa_{4}^{2} \lambda / 6, v_{A}(=$ $\left.\dot{R}_{A}\right)$ is the velocity of the apparent horizon, and $f_{A}\left(=\dot{v}_{A}\right)$ is the acceleration of the apparent horizon.

Due to the energy conservation relation for matter (i.e., (11)) and from the Bianchi identity we obtain

$$
\dot{\rho}_{t}+3 H\left(\rho_{t}+p_{t}\right)=0 .
$$

As a result the effective pressure and effective energy density also satisfy the conservation relation

$$
\dot{\rho}_{e}+3 H\left(\rho_{e}+p_{e}\right)=0 .
$$

Here the work density term can be broken up in the following form:

$$
W=W_{m}+W_{e},
$$

with

$$
\begin{aligned}
W_{m} & =\frac{\rho-p}{2}, \\
W_{e} & =-\frac{\kappa_{5}^{4} \rho p}{12} .
\end{aligned}
$$

Also, the energy supply vector can be decomposed as

$$
\psi=\psi_{m}+\psi_{e}
$$

with

$$
\begin{aligned}
& \psi_{m}=-\frac{1}{2}(\rho+p) H R d t+\frac{1}{2}(\rho+p) a d r \\
& \psi_{e}=-\frac{\kappa_{5}^{4}}{12} \rho(\rho+p) H R d t+\frac{\kappa_{5}^{4}}{12} \rho(\rho+p) a d r .
\end{aligned}
$$

As light rays move along the radial direction that is normal to the surface of the event horizon and we have $\partial \xi^{ \pm}=\partial_{t} \mp a \partial_{r}$ as one form along the normal direction, $\partial_{ \pm}=-\sqrt{2}\left(\partial_{t} \mp(1 / a) \partial_{r}\right)$ may be chosen along the tangential direction to the surface of the event horizon. Thus we choose [38]

$$
\xi_{E}=\partial_{t}-\frac{1}{a} \partial_{r},
$$

as the tangential vector to the surface of the event horizon.
Now, projecting the unified first law along $\xi_{E}$, the first law of thermodynamics of the event horizon is obtained as [3234]

$$
\left\langle d E, \xi_{E}\right\rangle=\kappa_{E}\left\langle d A, \xi_{E}\right\rangle+\left\langle W d V, \xi_{E}\right\rangle .
$$

Note that the pure matter energy supply $A \psi_{m}$, when projected on the event horizon, gives the heat flow $\delta Q$ in the Clausius relation $\delta Q=T d S$. Hence from (22), we have

$$
\delta Q=\left\langle A \psi_{m}, \xi_{E}\right\rangle=\kappa_{E}\left\langle d A, \xi_{E}\right\rangle-\left\langle A \psi_{e}, \xi_{E}\right\rangle .
$$

Using (13), (14), and (20) we obtain (after a simple algebra)

$$
\left\langle A \psi_{m}, \xi_{E}\right\rangle=\kappa_{E} R_{E} \dot{R}_{E}+\frac{A\left(H R_{E}+1\right) \rho(\rho+p) \kappa_{5}^{4}}{12} .
$$

Now using the extended Hawking temperature on event horizon, the above equation can be written as

$$
\begin{aligned}
& \left\langle A \psi_{m}, \xi_{E}\right\rangle \\
& =T\left\langle\frac{R_{E} d R_{E}}{4}-\frac{\kappa_{5}^{4}}{96} \frac{R_{A}^{2} R_{E}}{1-\epsilon}\left(H R_{E}+1\right) \rho(\rho+p) d t, \xi_{E}\right\rangle .
\end{aligned}
$$

Thus comparing with Clausius relation $\delta Q=T d S$ and integrating we have the entropy on the event horizon:

$$
S_{E}=\frac{A_{E}}{4}-\frac{\kappa_{5}^{4}}{96} \int\left(\frac{R_{A}^{2} R_{E}}{1-\epsilon}\right)\left(\frac{H R_{E}+1}{H R_{E}-1}\right) \rho(\rho+p) d R_{E} .
$$

Similarly, for apparent horizon, considering [32]

$$
\xi_{A}=\partial_{t}-(1-2 \epsilon) H r \partial_{r}
$$

as the tangent vector to the surface of the apparent horizon, the expression for entropy becomes

$$
S_{A}=\frac{A_{A}}{4}-\frac{\kappa_{5}^{4}}{96} \int \frac{R_{A}^{3}}{\epsilon} \rho(\rho+p) d R_{A} .
$$

We will now examine the thermodynamic inequalities (8) for (a) perfect fluid and (b) Modified Chaplygin Gas.

(a) Perfect Fluid. The equation of state parameter $\omega(<-1 / 3)$ is assumed to be constant. For this simple fluid, the horizon entropies take the forms

$$
\begin{aligned}
S_{E}= & \frac{A_{E}}{4}-\frac{\kappa_{5}^{4} \rho_{0}^{2}(\omega+1)}{96} \\
& \times \int\left(\frac{R_{A}^{2} R_{E}}{1-\epsilon}\right)\left(\frac{H R_{E}+1}{H R_{E}-1}\right)\left(\frac{1}{a^{3(\omega+1)}}\right)^{2} d R_{E}, \\
S_{A}= & \frac{A_{A}}{4}-\frac{\kappa_{5}^{4} \rho_{0}^{2}(\omega+1)}{96} \int \frac{R_{A}^{3}}{\epsilon}\left(\frac{1}{a^{3(\omega+1)}}\right)^{2} d R_{A},
\end{aligned}
$$

where $\rho=\rho_{0} / a^{3(\omega+1)}$ and $\rho_{0}$ is an arbitrary constant. 
Hence the time derivative of the total entropy (i.e., entropy of the horizon + entropy of the fluid) is given by

$$
\begin{aligned}
& \dot{S}_{T A}=\frac{R_{A} v_{A}}{4}-\frac{R_{A}^{3}}{8}\left(\rho_{e}+p_{e}\right)+\frac{R_{A} v_{A}\left(v_{A}-1\right)}{2\left(2-v_{A}\right)}, \\
& \text { for apparent horizon, } \\
& \dot{S}_{T E}=\frac{R_{E} v_{E}}{4}-\frac{R_{A}^{2} R_{E}}{2\left(2-v_{A}\right)}\left\{\frac{v_{E}+2}{4}\left(\rho_{e}+p_{e}\right)+v_{A} H^{2}\right\},
\end{aligned}
$$

for event horizon.

Again, taking the time derivative of $\dot{S}_{T A}$ and $\dot{S}_{T E}$ we have

$$
\begin{aligned}
\ddot{S}_{T A}= & \frac{R_{A} f_{A}}{4}\left\{1-\frac{2}{\left(v_{A}-2\right)^{2}}\left(v_{A}^{2}-4 v_{A}+2\right)\right\} \\
& -\frac{R_{A}^{2}}{8}\left\{3 v_{A}\left(\rho_{e}+p_{e}\right)+8 R_{A} \frac{\partial\left(\rho_{e}+p_{e}\right)}{\partial t}\right\} \\
& -\frac{v_{A}^{2}\left(v_{A}-1\right)}{2\left(v_{A}-2\right)}, \text { for apparent horizon, } \\
\ddot{S}_{T E}= & \frac{R_{E} f_{E}\left\{1-\frac{R_{A}^{2}\left(\rho_{e}+p_{e}\right)}{2\left(2-v_{A}\right)}\right\}+\frac{v_{E}^{2}}{4}-\frac{R_{A}^{2} R_{E}}{8\left(2-v_{A}\right)}}{4} \\
& \times\left\{\left(v_{E}+2\right)\left(2 \frac{v_{A}}{R_{A}}+\frac{v_{E}}{R_{E}}+\frac{f_{A}}{2-v_{A}}\right)\left(\rho_{e}+p_{e}\right)\right. \\
& \left.+\frac{\partial\left(\rho_{e}+p_{e}\right)}{\partial t}\right\} \\
& +4 v_{A} H^{2}\left\{\frac{v_{E}}{R_{E}}+\frac{2 f_{A}}{v_{A}\left(2-v_{A}\right)}\right\}, \text { for event horizon. }
\end{aligned}
$$

In (30)-(31), $\rho_{e}+p_{e}=\left(\kappa_{5}^{4} / 9\right)(1+\omega) \rho^{2},(\partial / \partial t)\left(\rho_{e}+p_{e}\right)=$ $\left(\kappa_{5}^{4} / 9\right)\left(f_{A}-4 H v_{A}\right) \rho^{2}$, and $v_{E}\left(=\dot{R}_{E}\right)$ and $f_{E}\left(=\dot{v}_{E}\right)$ are, respectively, the velocity and the acceleration of the event horizon. Due to complicated expressions we cannot infer the sign of the above expressions, so we examine the validity of GSLT and TE graphically in Figures 1 and 2 for $H=1, \kappa_{5}=1$, and $R_{E}=3$. Also restrictions on $\omega$ to satisfy inequalities (8) are shown in Table 1.

(b) Modified Chaplygin Gas. The equation of state for Modified Chaplygin Gas is written as $[41,50]$

$$
p=\gamma \rho-\frac{B}{\rho^{n}}
$$

where $\gamma(\leq 1)$ and $B, n$ are positive constants. Now solving the energy conservation equation (16), we have

$$
\rho^{n+1}=\frac{1}{\gamma+1}\left[B+\left(\frac{C}{a^{3}}\right)^{(\gamma+1)(n+1)}\right],
$$

where $C$ is an arbitrary constant.

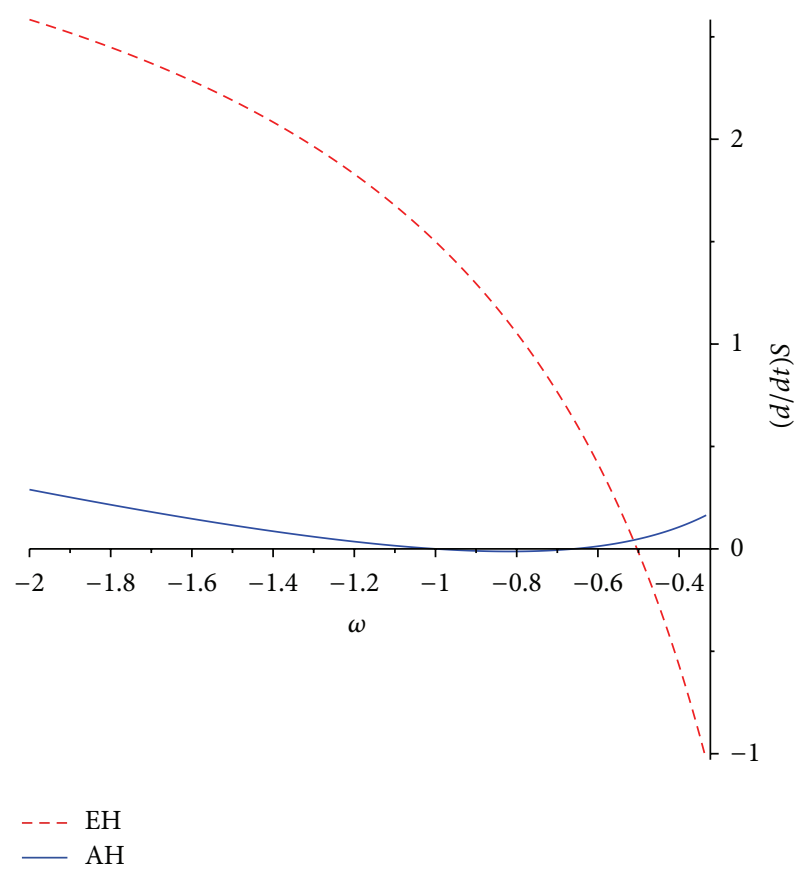

FIGURE 1: GSLT for RSII brane for perfect fluid with constant equation of state.

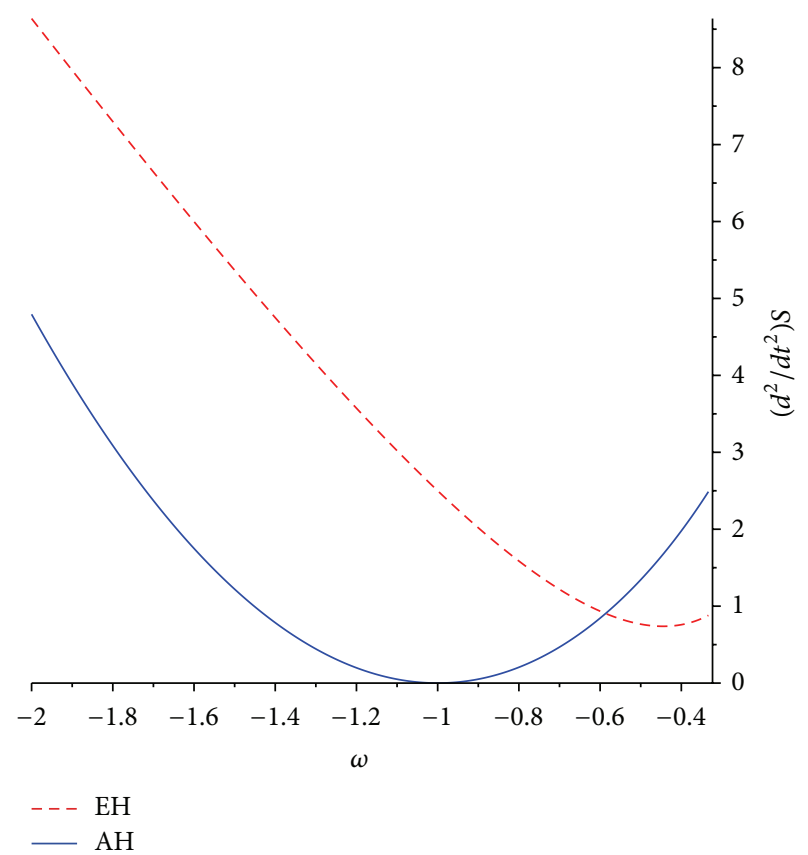

FIGURE 2: TE for RSII brane for perfect fluid with constant equation of state.

In this model the velocity of the apparent horizon is $v_{A}=$ $3 C(1+\gamma) / 2\left(B a^{\mu}+C\right), \mu=3(1+n)(1+\gamma)$. The radius of the event horizon can be expressed in terms of hypergeometric function as

$$
R_{E}=R_{12} F_{1}\left[\frac{1}{2(n+1)}, \frac{1}{\mu}, 1+\frac{1}{\mu}, \frac{-C}{B a^{\mu}}\right],
$$

where $R_{1}=\sqrt{3}(1+\gamma)^{1 / 2(n+1)} / B^{1 / 2(n+1)}$. 
TABLE 1: Conditions for GSLT and TE to hold when Universe is filled with perfect fluid having constant equation of state.

\begin{tabular}{lcc}
\hline Horizons in brane world & GSLT & TE \\
\hline AH for RSII brane & $\omega \geq-0.64$ or & Does not hold \\
EH for RSII brane & $\omega \leq-0.98$ & \\
& $\omega \leq-0.51$ & Does not hold \\
AH for DGP brane & $\omega \geq-0.82$ or & $\omega<-1.08$ or \\
EH for DGP brane & $\omega \leq-1$ & $-0.89<\omega<-0.54$ \\
\end{tabular}

Hence expressions for horizon entropy become

$$
\begin{aligned}
S_{E}= & \frac{A_{E}}{4}-\frac{\kappa_{5}^{4}}{96(\gamma+1)^{2 /(n+1)}} \\
& \times \int\left(\frac{R_{A}^{2} R_{E}}{1-\epsilon}\right)\left(\frac{H R_{E}+1}{H R_{E}-1}\right)\left[B+\left(\frac{C}{a^{3}}\right)^{(\gamma+1)(n+1)}\right]^{2 /(n+1)} \\
& \times\left[\gamma+1-B(\gamma+1)\left\{B+\left(\frac{C}{a^{3}}\right)^{(\gamma+1)(n+1)}\right\}^{-1}\right] d R_{E},
\end{aligned}
$$$$
S_{A}=\frac{A_{A}}{4}-\frac{\kappa_{5}^{4}}{96(\gamma+1)^{2 /(n+1)}}
$$$$
\times \int \frac{R_{A}^{3}}{\epsilon}\left[B+\left(\frac{C}{a^{3}}\right)^{(\gamma+1)(n+1)}\right]^{2 /(n+1)}
$$$$
\times\left[\gamma+1-B(\gamma+1)\left\{B+\left(\frac{C}{a^{3}}\right)^{(\gamma+1)(n+1)}\right\}^{-1}\right] d R_{A} .
$$

Now, the time derivative of the total entropy is given by

$$
\begin{aligned}
& \dot{S}_{T A}=\frac{R_{A} v_{A}}{4}-\frac{R_{A}^{3}}{8}\left(\rho_{e}+p_{e}\right)+\frac{R_{A} v_{A}\left(v_{A}-1\right)}{2\left(2-v_{A}\right)}, \\
& \text { for apparent horizon, } \\
& \dot{S}_{T E}=\frac{R_{E} v_{E}}{4}-\frac{R_{A}^{2} R_{E}}{2\left(2-v_{A}\right)}\left\{\frac{v_{E}+2}{4}\left(\rho_{e}+p_{e}\right)+v_{A} H^{2}\right\},
\end{aligned}
$$

for event horizon.

Again, taking the derivative of $\dot{S}_{T A}$ and $\dot{S}_{T E}$ we have

$$
\begin{aligned}
\ddot{S}_{T A}= & \frac{R_{A} f_{A}}{4}\left\{1-\frac{2}{\left(v_{A}-2\right)^{2}}\left(v_{A}^{2}-4 v_{A}+2\right)\right\} \\
& -\frac{R_{A}^{2}}{8}\left\{3 v_{A}\left(\rho_{e}+p_{e}\right)+8 R_{A} \frac{\partial\left(\rho_{e}+p_{e}\right)}{\partial t}\right\} \\
& -\frac{v_{A}^{2}\left(v_{A}-1\right)}{2\left(v_{A}-2\right)}, \quad \text { for apparent horizon, }
\end{aligned}
$$

$$
\begin{aligned}
\ddot{S}_{T E}= & \frac{R_{E} f_{E}}{4}\left\{1-\frac{R_{A}^{2}\left(\rho_{e}+p_{e}\right)}{2\left(2-v_{A}\right)}\right\}+\frac{v_{E}^{2}}{4}-\frac{R_{A}^{2} R_{E}}{8\left(2-v_{A}\right)} \\
& \times\left\{\left(v_{E}+2\right)\left(2 \frac{v_{A}}{R_{A}}+\frac{v_{E}}{R_{E}}+\frac{f_{A}}{2-v_{A}}\right)\left(\rho_{e}+p_{e}\right)\right. \\
& \left.+\frac{\partial\left(\rho_{e}+p_{e}\right)}{\partial t}\right\}+4 v_{A} H^{2}\left\{\frac{v_{E}}{R_{E}}+\frac{2 f_{A}}{v_{A}\left(2-v_{A}\right)}\right\},
\end{aligned}
$$

for event horizon.

In (36)-(37), $\rho_{e}+p_{e}=\left(\kappa_{5}^{4} / 9\right) \rho^{2}\left(1+\gamma-B / \rho^{n+1}\right)$ and $(\partial / \partial t)\left(\rho_{e}+\right.$ $\left.p_{e}\right)=\left(\kappa_{5}^{4} / 9\right)\left(f_{A}-4 H v_{A}\right) \rho^{2}$, where $\rho$ is given by (33).

As before, due to complicated form of the above entropy variations, the thermodynamical inequalities (8) are examined graphically in Figures 3 and 4 . In particular $\dot{S}_{T A}, \ddot{S}_{T A}, \dot{S}_{T E}$, and $\ddot{S}_{T E}$ are plotted against $\gamma$ in the figures considering $H=1$, $\kappa_{5}=1, a=1, n=0.25, B=2$, and $C=1$. Also in Table 2 bounds on $\gamma$ are shown to satisfy inequalities (8).

DGP Brane World. In a flat, homogeneous, and isotropic brane, the Friedmann equation in DGP model is given by $[51,52]$

$$
H^{2}-\widetilde{\epsilon} \frac{H}{r_{c}}=\frac{\rho}{3}
$$

where $r_{c}$ is the crossover scale which determines the transition from $4 \mathrm{D}$ to $5 \mathrm{D}$ behavior and $\widetilde{\epsilon}= \pm 1$ corresponds to standard DGP $(+)$ model (self-accelerating without any form of dark energy) and DGP(-) model (not self-accelerating and requiring dark energy), respectively.

From (38) and using the conservation equation (16), it can be shown that

$$
\dot{H}=-\frac{1}{2}\left[\rho+p+\frac{\widetilde{\epsilon}(\rho+p)}{2 H r_{c}-\widetilde{\epsilon}}\right] .
$$

Thus we have

$$
\begin{gathered}
\rho_{e}+p_{e}=\frac{\widetilde{\epsilon}(\rho+p)}{2 H r_{c}-\widetilde{\epsilon}}, \\
\frac{\partial\left(\rho_{e}+p_{e}\right)}{\partial t}=\frac{2 \rho \widetilde{\epsilon}}{3\left(2 H r_{c}-\widetilde{\epsilon}\right)}\left(f_{A}-2 H v_{A}^{2}+\frac{2 H^{2} v_{A}^{2} r_{c}}{2 H r_{c}-\widetilde{\epsilon}}\right) .
\end{gathered}
$$

Considering $\xi$ as given by (27) (for apparent horizon) and by (21) (for event horizon) and proceeding in the same way as before, the expressions of entropy on the horizon (apparent/event) for the validity of the unified first law are given by

$$
\begin{aligned}
& S_{A}=\frac{A_{A}}{4}-\frac{1}{16} \widetilde{\epsilon} \int\left(\frac{R_{A}^{3}}{\epsilon}\right)\left(\frac{\rho+p}{2 H r_{c}-\widetilde{\epsilon}}\right) d R_{A}, \\
& S_{E}=\frac{A_{E}}{4}-\frac{1}{16} \widetilde{\epsilon} \int\left(\frac{R_{A}^{2} R_{E}}{1-\epsilon}\right)\left(\frac{H R_{E}+1}{H R_{E}-1}\right) \frac{\rho+p}{2 H r_{c}-\widetilde{\epsilon}} d R_{E} .
\end{aligned}
$$




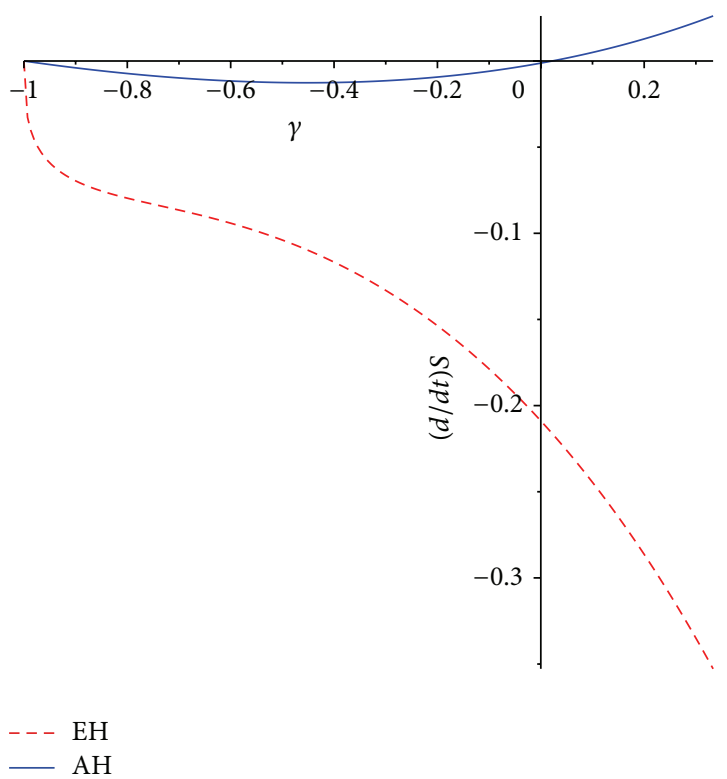

FIgURE 3: GSLT for RSII brane for Modified Chaplygin Gas.

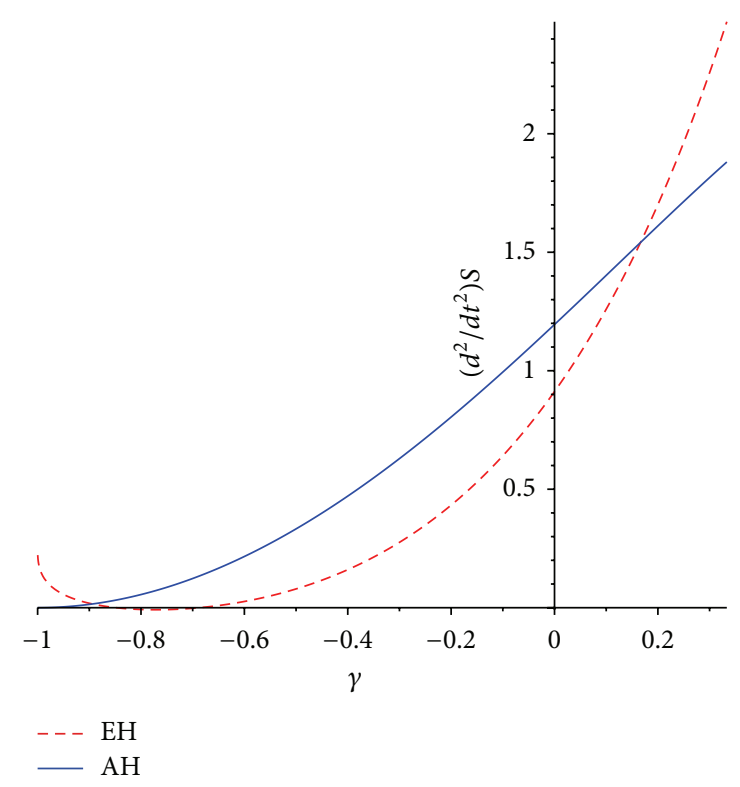

FIGURE 4: TE for RSII brane for Modified Chaplygin Gas.

TABLE 2: Conditions for GSLT and TE to hold when Universe is filled with Modified Chaplygin Gas.

\begin{tabular}{lcc}
\hline Horizons in brane world & GSLT & TE \\
\hline AH for RSII brane & $\gamma \geq 0.02$ & Does not hold \\
EH for RSII brane & Does not hold & $-0.85<\gamma<-0.68$ \\
AH for DGP brane & $\gamma=-1$ or & $\gamma>0.20$ \\
& $\gamma \geq-0.529$ & \\
EH for DGP brane & $\gamma=-1$ or & $-0.95<\gamma<-0.74$ \\
\hline
\end{tabular}

As in RSII brane we take the time derivative of fluid entropy from Gibb's equation and, combining with the time derivative of the horizon entropy expressions, we have

$$
\begin{aligned}
& \dot{S}_{T A}=\frac{R_{A} v_{A}}{4}-\frac{R_{A}^{3}}{8}\left(\rho_{e}+p_{e}\right)+\frac{R_{A} v_{A}\left(v_{A}-1\right)}{2\left(2-v_{A}\right)}, \\
& \text { for apparent horizon, } \\
& \dot{S}_{T E}=\frac{R_{E} v_{E}}{4}-\frac{R_{A}^{2} R_{E}}{2\left(2-v_{A}\right)}\left\{\frac{v_{E}+2}{4}\left(\rho_{e}+p_{e}\right)+v_{A} H^{2}\right\},
\end{aligned}
$$

for event horizon.

Again, taking the time derivative of $\dot{S}_{T A}$ and $\dot{S}_{T E}$ we have

$$
\begin{aligned}
\ddot{S}_{T A}= & \frac{R_{A} f_{A}}{4}\left\{1-\frac{2}{\left(v_{A}-2\right)^{2}}\left(v_{A}^{2}-4 v_{A}+2\right)\right\}-\frac{R_{A}^{2}}{8} \\
& \times\left\{3 v_{A}\left(\rho_{e}+p_{e}\right)+8 R_{A} \frac{\partial\left(\rho_{e}+p_{e}\right)}{\partial t}\right\}-\frac{v_{A}^{2}\left(v_{A}-1\right)}{2\left(v_{A}-2\right)},
\end{aligned}
$$

for apparent horizon,

$$
\begin{aligned}
\ddot{S}_{T E}= & \frac{R_{E} f_{E}}{4}\left\{1-\frac{R_{A}^{2}\left(\rho_{e}+p_{e}\right)}{2\left(2-v_{A}\right)}\right\}+\frac{v_{E}^{2}}{4}-\frac{R_{A}^{2} R_{E}}{8\left(2-v_{A}\right)} \\
& \times\left\{\left(v_{E}+2\right)\left(2 \frac{v_{A}}{R_{A}}+\frac{v_{E}}{R_{E}}+\frac{f_{A}}{2-v_{A}}\right)\left(\rho_{e}+p_{e}\right)\right. \\
& \left.+\frac{\partial\left(\rho_{e}+p_{e}\right)}{\partial t}\right\} \\
& +4 v_{A} H^{2}\left\{\frac{v_{E}}{R_{E}}+\frac{2 f_{A}}{v_{A}\left(2-v_{A}\right)}\right\}, \text { for event horizon. }
\end{aligned}
$$

In (42)-(43), $\rho_{e}+p_{e}$ and $(\partial / \partial t)\left(\rho_{e}+p_{e}\right)$ are to be substituted from (40).

For perfect fluid with constant equation of state, the expressions of horizon entropy become

$$
\begin{aligned}
S_{A}= & \frac{A_{A}}{4}-\frac{1}{16} \widetilde{\epsilon}(\omega+1) \rho_{0} \\
& \times \int\left(\frac{R_{A}^{3}}{\epsilon}\right)\left(\frac{1}{2 H r_{c}-\widetilde{\epsilon}}\right) \frac{1}{a^{3(\omega+1)}} d R_{A}, \\
S_{E}= & \frac{A_{E}}{4}-\frac{1}{16} \widetilde{\epsilon}(\omega+1) \rho_{0} \\
& \times \int\left(\frac{R_{A}^{2} R_{E}}{1-\epsilon}\right)\left(\frac{H R_{E}+1}{H R_{E}-1}\right)\left(\frac{1}{2 H r_{c}-\widetilde{\epsilon}}\right) \frac{1}{a^{3(\omega+1)}} d R_{E} .
\end{aligned}
$$

Similar to RSII model, $\dot{S}_{T A}, \ddot{S}_{T A}, \dot{S}_{T E}$, and $\ddot{S}_{T E}$ have been plotted against $\omega$ for apparent horizon $(\mathrm{AH})$ and event horizon $(\mathrm{EH})$ in Figures 5 and 6, respectively, considering $H=1.5, \epsilon=1, R_{E}=3$, and $r_{c}=2$. 


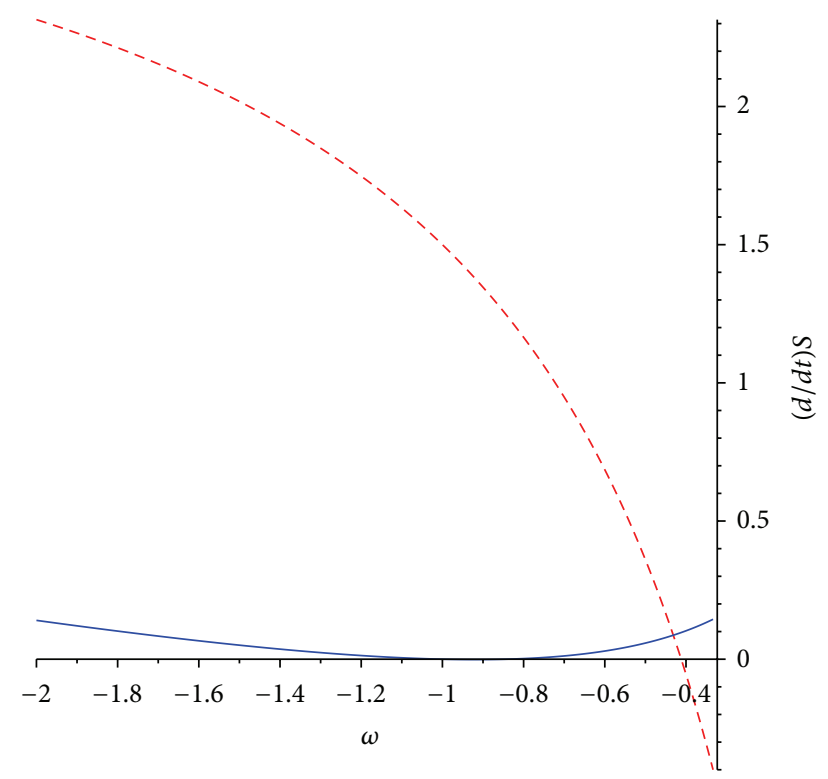

- - $\mathrm{EH}$

$-\mathrm{AH}$

FIGURE 5: GSLT for DGP brane for perfect fluid with constant equation of state.

For Modified Chaplygin Gas, the horizon entropy takes the forms

$$
\begin{aligned}
S_{A}=\frac{A_{A}}{4} & -\frac{1}{16(\gamma+1)^{1 /(n+1)} \widetilde{\epsilon}} \\
\times & \int\left(\frac{R_{A}^{3}}{\epsilon}\right)\left(\frac{1}{2 H r_{c}-\widetilde{\epsilon}}\right)\left[B+\left(\frac{C}{a^{3}}\right)^{(\gamma+1)(n+1)}\right]^{1 /(n+1)} \\
& \times\left[\gamma+1-B(\gamma+1)\left\{B+\left(\frac{C}{a^{3}}\right)^{(\gamma+1)(n+1)}\right\}^{-1}\right] d R_{A}, \\
S_{E}=\frac{A_{E}}{4} & \left.-\frac{1}{16(\gamma+1)^{1 /(n+1)} \widetilde{\epsilon}}\right] \\
\times & \left(\frac{R_{A}^{2} R_{E}}{1-\epsilon}\right)\left(\frac{H R_{E}+1}{H R_{E}-1}\right)\left(\frac{1}{2 H r_{c}-\widetilde{\epsilon}}\right) \\
& \left.\left.\times\left[B+\left(\frac{C}{a^{3}}\right)^{(\gamma+1)(n+1)}\right]^{1 /(n+1)}\right\}^{-1}\right] d R_{E} . \\
& \times\left[\gamma+1-B(\gamma+1)\left\{B+\left(\frac{C}{a^{3}}\right)^{(\gamma+1)(n+1)}\right\}^{-1}\right.
\end{aligned}
$$

We have plotted $\dot{S}_{T A}, \ddot{S}_{T A}, \dot{S}_{T E}$, and $\ddot{S}_{T E}$ against $\gamma$ for apparent horizon $(\mathrm{AH})$ and event horizon $(\mathrm{EH})$ in Figures 7 and 8, respectively, considering $H=1.5, \epsilon=1, a=1$, $n=0.25, B=2, C=1$, and $r_{c}=2$.

Thus, in the present work, we have considered Universal thermodynamics for brane world scenario both in RSII model

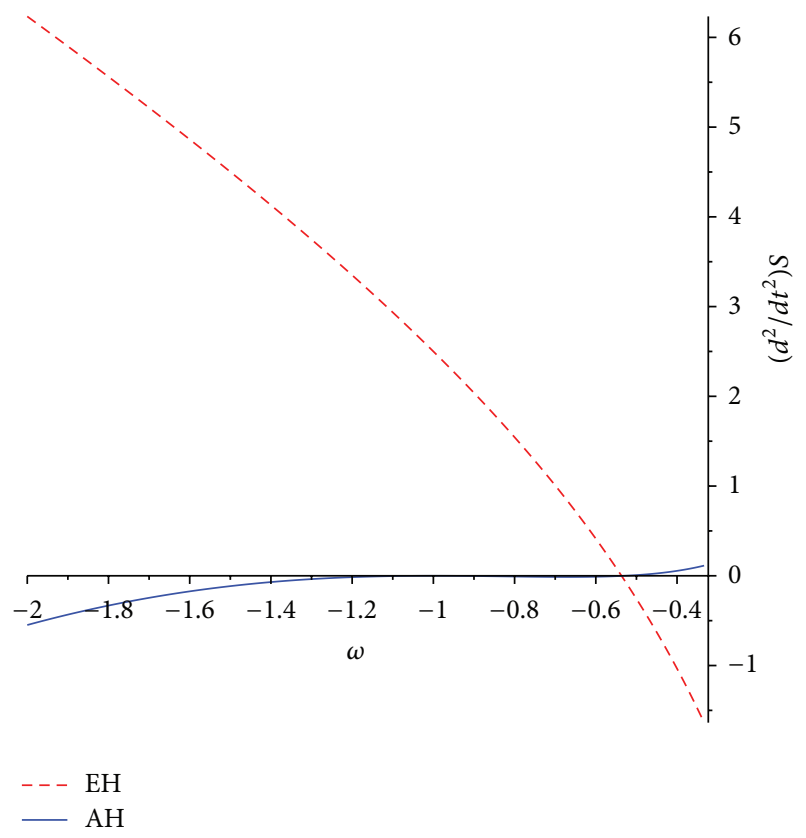

FIGURE 6: TE for DGP brane for perfect fluid with constant equation of state.

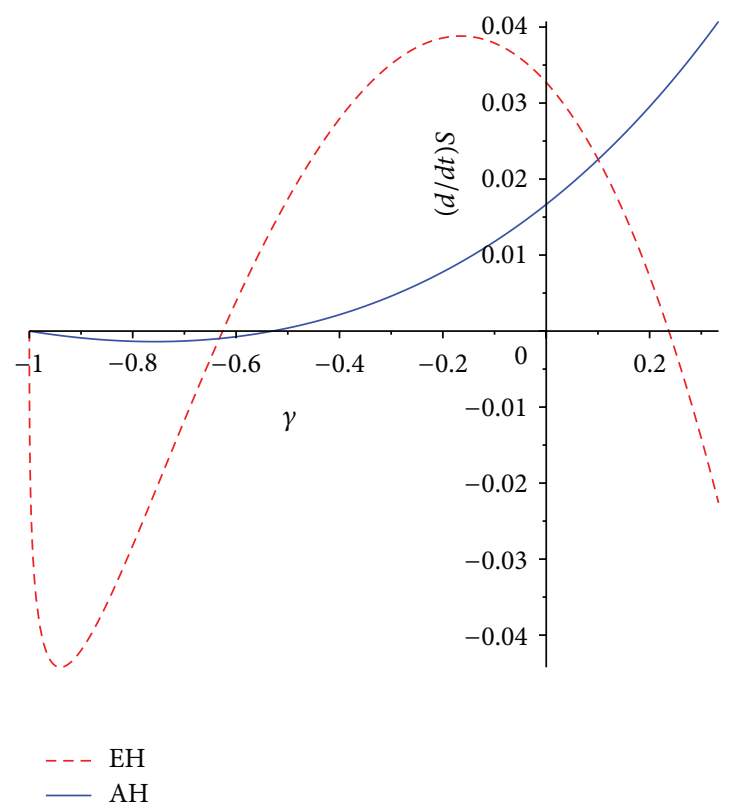

FIGURE 7: GSLT for DGP brane for Modified Chaplygin Gas.

and in DGP model when the FRW Universe is bounded by the horizon (event/apparent). The matter content in the Universe is chosen as one of the following fluids:

(i) perfect fluid with constant equation of state which may be considered as normal fluid or an exotic fluid depending on the equation of state parameter,

(ii) Modified Chaplygin Gas model which is a unified model of dark matter and dark energy, extending up to $\Lambda \mathrm{CDM}$. 


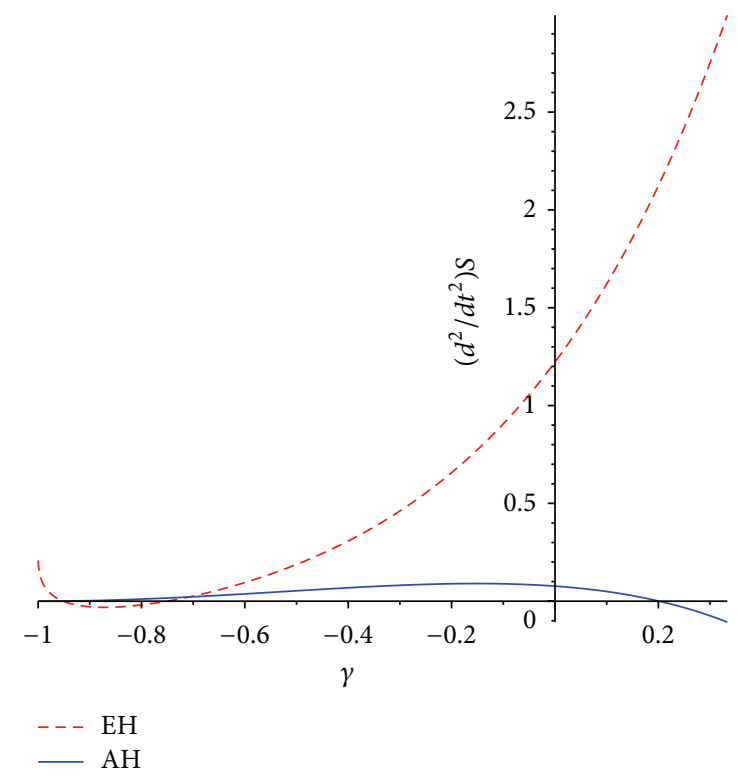

Figure 8: TE for DGP brane for Modified Chaplygin Gas.

The temperature of the fluid as well as that of the horizon is taken as extended Hawking temperature and we have examined the validity of the unified first law on the horizon. It turns out that the entropy on the horizon is no longer the Bekenstein entropy; rather the correction term is in integral form.

From the figures as well as from the tables we see that GSLT holds for both horizons and for both fluids with some restrictions on the equation of state parameter " $\omega$ " or on the parameter " $\gamma$ " (for MCG) except that GSLT does not hold at all at the event horizon for RSII brane with MCG model. On the other hand, thermodynamical equilibrium does not hold at the apparent horizon for RSII brane model for both fluids while for event horizon in RSII brane model TE does not hold for perfect fluid and it holds for MCG in a restricted range of " $\gamma$ " (see Table 2).

For DGP brane model, TE as well as GSLT holds for both brane models and for both fluids in some restricted range of the parameters $\gamma$ and $\omega$. So, from the above thermodynamical analysis, we cannot conclude that one horizon is more favourable than the other.

From the field equations (12), using (13), we see that the Hubble parameter depends on the brane tension and the effect of the higher dimension in RSII brane model. Similarly, for DGP brane scenario the field equations (38) and (39) depend on the crossover scale $\left(r_{c}\right)$ which determines the transition from 4-dimensional to 5-dimensional behavior. As $R_{A}\left(=1 / \sqrt{H^{2}+k / a^{2}}\right)$ and $R_{E}\left(=a \int_{a}^{\infty}\left(d a / a^{2} H\right)\right)$ depend on the Hubble parameter, the radius of horizons depends on the five-dimensional effect. In the present work, the factors $\kappa_{5}^{4}$ in RSII brane model and " $r_{c}$ " in DGP model represent the effect of the corresponding brane model.

Finally, it should be noted that in earlier works in this direction by Cai et al. [32-34] it was concluded that Universal thermodynamics in these modified gravity theories is not equilibrium in nature and there is entropy production term for the validity of unified first law. On the contrary, in the present work we have shown that Universal thermodynamics remains equilibrium in nature in modified gravity theories but the Bekenstein entropy needs some correction term. Therefore, we conclude that one should consider corrections to Bekenstein entropy so that Universal thermodynamics in modified gravity theories still remains equilibrium in nature.

\section{Conflict of Interests}

The authors declare that there is no conflict of interests regarding the publication of this paper.

\section{Acknowledgments}

The author Saugata Mitra is thankful to UGC for NETJRF. The author Subhajit Saha is thankful to UGC-BSR Programme of Jadavpur University for awarding Research Fellowship. Subenoy Chakraborty is thankful to UGC-DRS Programme, Department of Mathematics, Jadavpur University.

\section{References}

[1] A. G. Riess, A. V. Filippenko, P. Challis et al., "Observational evidence from supernovae for an accelerating universe and a cosmological constant," The Astronomical Journal, vol. 116, no. 3, p. 1009, 1998.

[2] S. Perlmutter, G. Aldering, G. Goldhaber et al., "Measurements of $\Omega$ and $\Lambda$ from 42 high-redshift supernovae," The Astrophysical Journal, vol. 517, no. 2, pp. 565-586, 1999.

[3] R. A. Knop, G. Aldering, R. Amanullah et al., "New constraints on $\Omega_{M}, \Omega_{\Lambda}$, and w from an independent set of 11 high-redshift supernovae observed with the hubble space telescope," The Astrophysical Journal, vol. 598, p. 102, 2003.

[4] P. de Bernardis, P. A. R. Ade, J. J. Bock et al., "A flat Universe from high-resolution maps of the cosmic microwave background radiation," Nature, vol. 404, no. 6781, pp. 955-959, 2000.

[5] D. N. Spergel, L. Verde, and H. V. Peiris, "First-year Wilkinson Microwave Anisotropy Probe (WMAP) observations: determination of cosmological parameters," The Astrophysical Journal Supplement Series, vol. 148, p. 175, 2003.

[6] T. Padmanabhan, "Cosmological constant-the weight of the vacuum," Physics Reports, vol. 380, no. 5-6, pp. 235-320, 2003.

[7] P. J. Peebles and B. Ratra, "The cosmological constant and dark energy," Reviews of Modern Physics, vol. 75, no. 2, pp. 559-606, 2003.

[8] C. G. Tsagas, A. Challinor, and R. Maartens, "Relativistic cosmology and large-scale structure," Physics Reports, vol. 465, no. 2-3, pp. 61-147, 2008.

[9] M. Li, X.-D. Li, S. Wang, and Y. Wang, "Dark energy," Communications in Theoretical Physics, vol. 56, no. 3, pp. 525-604, 2011.

[10] V. Sahni, "The cosmological constant problem and quintessence," Classical and Quantum Gravity, vol. 19, no. 13, pp. 34353448, 2002.

[11] E. J. Copeland, M. Sami, and S. Tsujikawa, "Dynamics of dark energy," International Journal of Modern Physics D, vol. 15, no. 11, pp. 1753-1935, 2006.

[12] T. Padmanabhan, "Dark energy and gravity," General Relativity and Gravitation, vol. 40, no. 2-3, pp. 529-564, 2008. 
[13] L. Randall and R. Sundrum, "Large mass hierarchy from a small extra dimension," Physical Review Letters, vol. 83, no. 17, pp. 3370-3373, 1999.

[14] L. Randall and R. Sundrum, "An alternative to compactification," Physical Review Letters, vol. 83, no. 23, pp. 4690-4693, 1999.

[15] R. Maartens, "Geometry and dynamics of the brane-world," http://arxiv.org/abs/gr-qc/0101059.

[16] G. Dvali, G. Gabadadze, and M. Porrati, "4D gravity on a brane in 5D Minkowski space," Physics Letters B, vol. 485, no. 1-3, pp. 208-214, 2000.

[17] D. Deffayet, "Cosmology on a brane in Minkowski bulk," Physics Letters B, vol. 502, pp. 199-208, 2001.

[18] C. Deffayet, G. Dvali, and G. Gabadadze, "Accelerated universe from gravity leaking to extra dimensions," Physical Review D, vol. 65, no. 4, Article ID 044023, 2002.

[19] E. Witten, "The cosmological constant from the viewpoint of string theory," http://arxiv.org/abs/hepph/0002297.

[20] S. W. Hawking, "Particle creation by black holes," Communications in Mathematical Physics, vol. 43, no. 3, pp. 199-220, 1975.

[21] J. D. Bekenstein, "Black holes and entropy," Physical Review D, vol. 7, pp. 2333-2346, 1973.

[22] J. M. Bardeen, B. Carter, and S. W. Hawking, "The four laws of black hole mechanics," Communications in Mathematical Physics, vol. 31, no. 2, pp. 161-170, 1973.

[23] J. Maldacena, "The large $N$ limit of superconformal field theories and supergravity," Advances in Theoretical and Mathematical Physics, vol. 2, no. 2, pp. 231-252, 1998.

[24] T. Jacobson, "Thermodynamics of spacetime: the Einstein equation of state," Physical Review Letters, vol. 75, no. 7, pp. 1260-1263, 1995.

[25] T. Padmanabhan, "Classical and quantum thermodynamics of horizons in spherically symmetric spacetimes," Classical and Quantum Gravity, vol. 19, no. 21, pp. 5387-5408, 2002.

[26] T. Padmanabhan, "Gravity and the thermodynamics of horizons," Physics Reports, vol. 406, no. 2, pp. 49-125, 2005.

[27] A. Paranjape, S. Sarkar, and T. Padmanabhan, "Thermodynamic route to field equations in Lanczos-LOVelock gravity," Physical Review D, vol. 74, no. 10, Article ID 104015, 9 pages, 2006.

[28] S. A. Hayward, "Gravitational energy in spherical symmetry," Physical Review D, vol. 53, no. 4, pp. 1938-1949, 1996.

[29] S. A. Hayward, "Unified first law of black-hole dynamics and relativistic thermodynamics," Classical and Quantum Gravity, vol. 15, no. 10, pp. 3147-3162, 1998.

[30] S. A. Hayward, "Energy conservation for dynamical black holes," Physical Review Letters, vol. 93, no. 25, Article ID 251101, 2004.

[31] S. A. Hayward, S. Mukhoyama, and M. C. Ashworth, "Dynamic black-hole entropy," Physics Letters A, vol. 256, no. 5-6, pp. 347350, 1999.

[32] R.-G. Cai and L.-M. Cao, "Unified first law and the thermodynamics of the apparent horizon in the FRW universe," Physical Review D: Particles, Fields, Gravitation, and Cosmology, vol. 75, no. 6, Article ID 064008, 11 pages, 2007.

[33] M. Akbar and R. G. Cai, "Thermodynamic behavior of the Friedmann equation at the apparent horizon of the FRW universe," Physical Review D, vol. 75, Article ID 084003, 2007.

[34] R. G. Cai and L. M. Cao, "Thermodynamics of apparent horizon in brane world scenario," Nuclear Physics B, vol. 785, no. 1-2, pp. 135-148, 2007.
[35] R. G. Cai and S. P. Kim, "First law of thermodynamics and Friedmann equations of Friedmann-Robertson-Walker universe," Journal of High Energy Physics, vol. 2005, article 050, 2005.

[36] C. Eling, R. Guedens, and T. Jacobson, "Nonequilibrium thermodynamics of spacetime," Physical Review Letters, vol. 96, no. 12, Article ID 121301, 2006.

[37] A. Sheykhi, B. Wang, and R.-G. Cai, "Thermodynamical properties of apparent horizon in warped DGP braneworld," Nuclear Physics B, vol. 779, no. 1-2, pp. 1-12, 2007.

[38] S. Mitra, S. Saha, and S. Chakraborty, "Universal thermodynamics in different gravity theories: modified entropy on the horizons," Physics Letters B, vol. 734, pp. 173-177, 2014.

[39] D. Bak and S. J. Rey, "Cosmic holography," Classical and Quantum Gravity, vol. 17, no. 15, pp. L83-L89, 2000.

[40] D. Pavón and W. Zimdahl, "A thermodynamic characterization of future singularities?" Physics Letters B, vol. 708, no. 3-5, pp. 217-220, 2012.

[41] I. Mocioiu and R. Roiban, "Dark energy from a renormalization group flow," Physical Review D, vol. 84, no. 4, Article ID 043512, 2014.

[42] N. Mazumder and S. Chakraborty, "Does the validity of the first law of thermodynamics imply that the generalized second law of thermodynamics of the universe is bounded by the event horizon?" Classical and Quantum Gravity, vol. 26, no. 19, Article ID 195016, 5 pages, 2009.

[43] N. Mazumder and S. Chakraborty, "Validity of the generalized second law of thermodynamics of the universe bounded by the event horizon in holographic dark energy model," General Relativity and Gravitation, vol. 42, pp. 813-820, 2010.

[44] N. Mazumder and S. Chakraborty, "Validity of the generalized second law of thermodynamics of the universe bounded by the event horizon in brane scenario," The European Physical Journal C, vol. 70, no. 1-2, pp. 329-334, 2010.

[45] S. Chakraborty, N. Mazumder, and R. Biswas, "The generalized second law of thermodynamics and the nature of entropy function," Europhysics Letters, vol. 91, no. 4, Article ID 40007, 2010.

[46] N. Mazumder, R. Biswas, and S. Chakraborty, "Thermodynamics of Lemaitre-Tolman-Bondi model," General Relativity and Gravitation, vol. 43, no. 6, pp. 1827-1836, 2011.

[47] J. Dutta and S. Chakraborty, "Validity of generalised second law in holographic DGP brane," General Relativity and Gravitation, vol. 42, no. 8, pp. 1863-1873, 2010.

[48] G. Izquierdo and D. Pavon, "Dark energy and the generalized second law," Physics Letters, B, vol. 633, no. 4-5, pp. 420-426, 2006.

[49] N. Mazumder and S. Chakraborty, "Validity of the generalized second law of thermodynamics of the universe bounded by the event horizon in brane scenario," The European Physical Journal C, vol. 70, no. 1, pp. 329-334, 2010.

[50] W. Chakraborty, U. Debnath, and S. Chakraborty, "Generalized cosmic chaplygin gas model with or without interaction," Gravitation and Cosmology, vol. 13, pp. 294-297, 2007.

[51] J. Dutta and S. Chakraborty, "Validity of generalised second law in holographic DGP brane," General Relativity and Gravitation, vol. 42, no. 8, pp. 1863-1873, 2010.

[52] J. Dutta and S. Chakraborty, "Generalized second law of thermodynamics for interacting dark energy in the DGP braneworld," International Journal of Theoretical Physics, vol. 50, no. 8, pp. 2383-2390, 2011. 

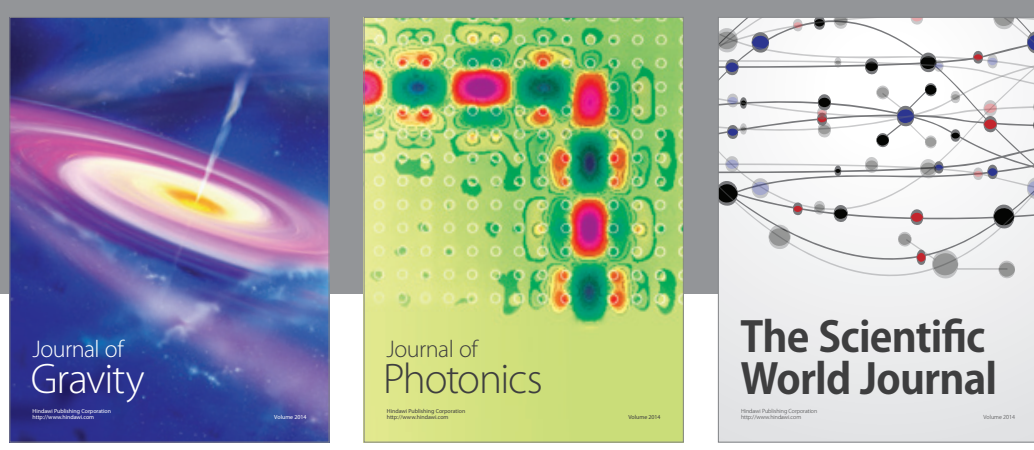

The Scientific World Journal
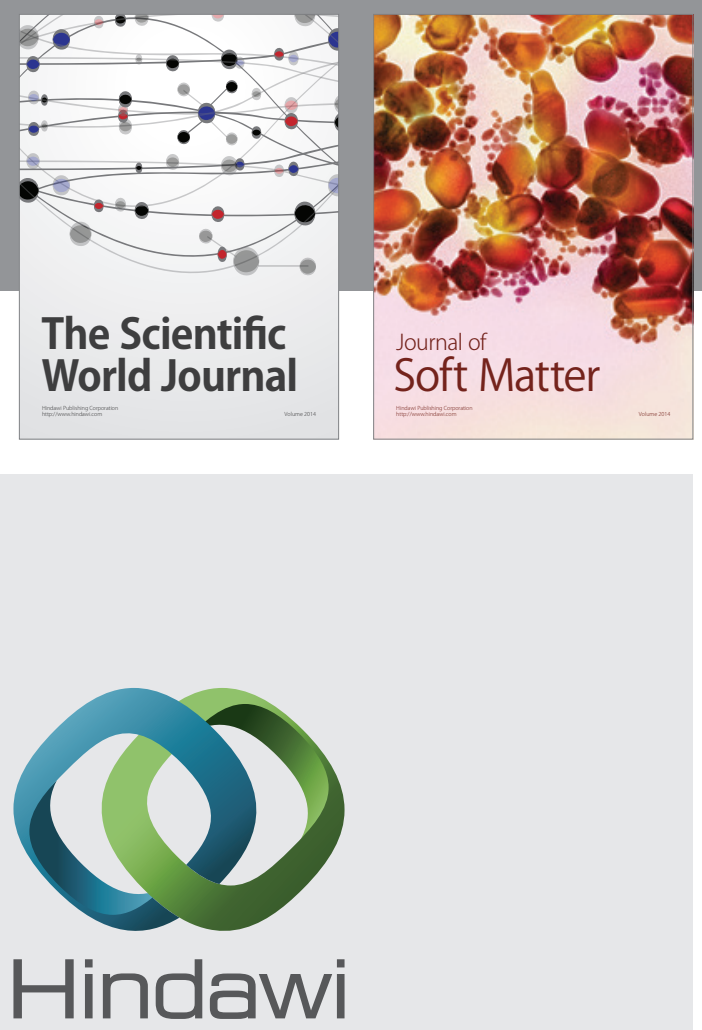

Submit your manuscripts at

http://www.hindawi.com

nternational Journal of

Statistical Mechanics
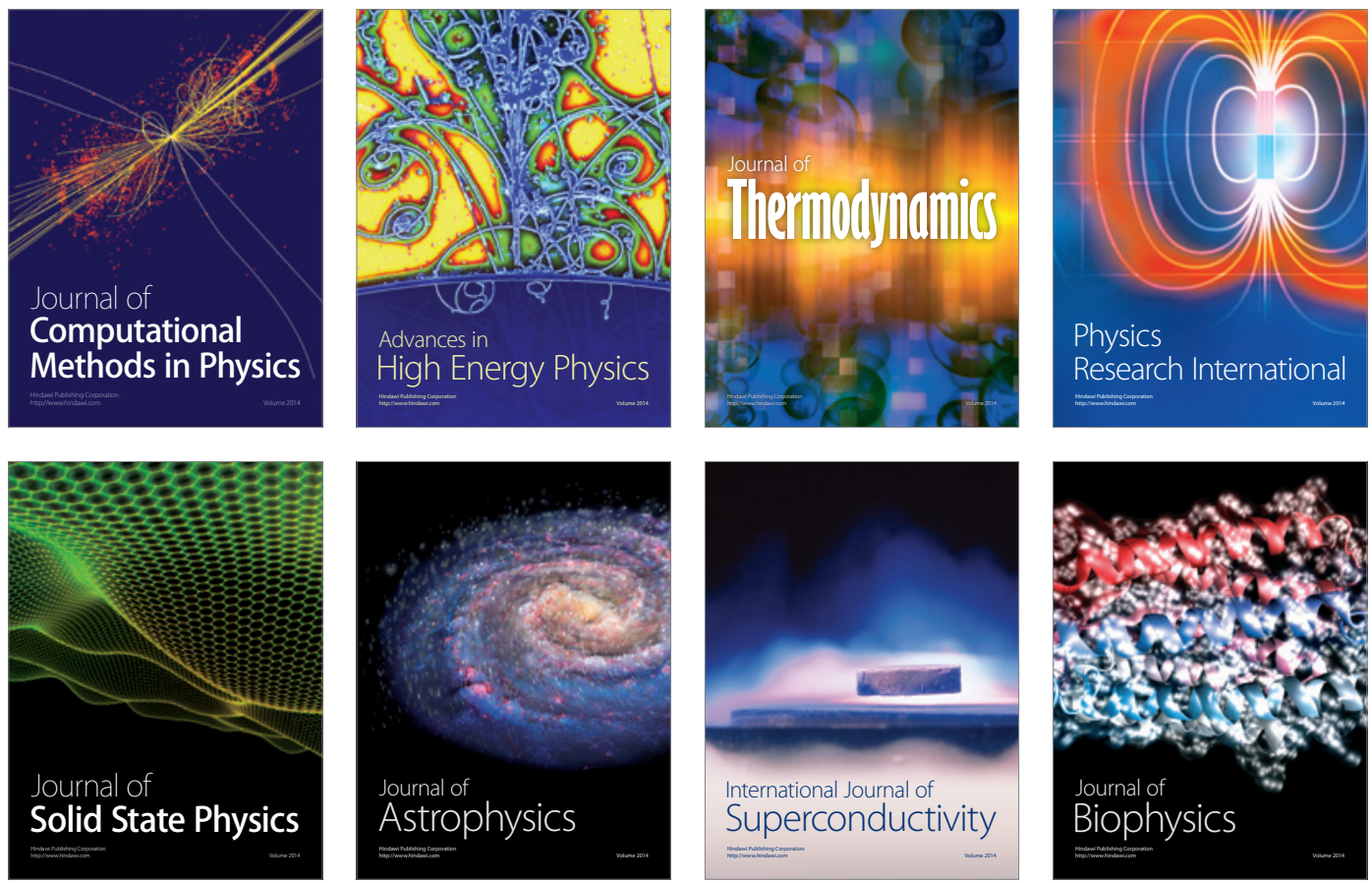
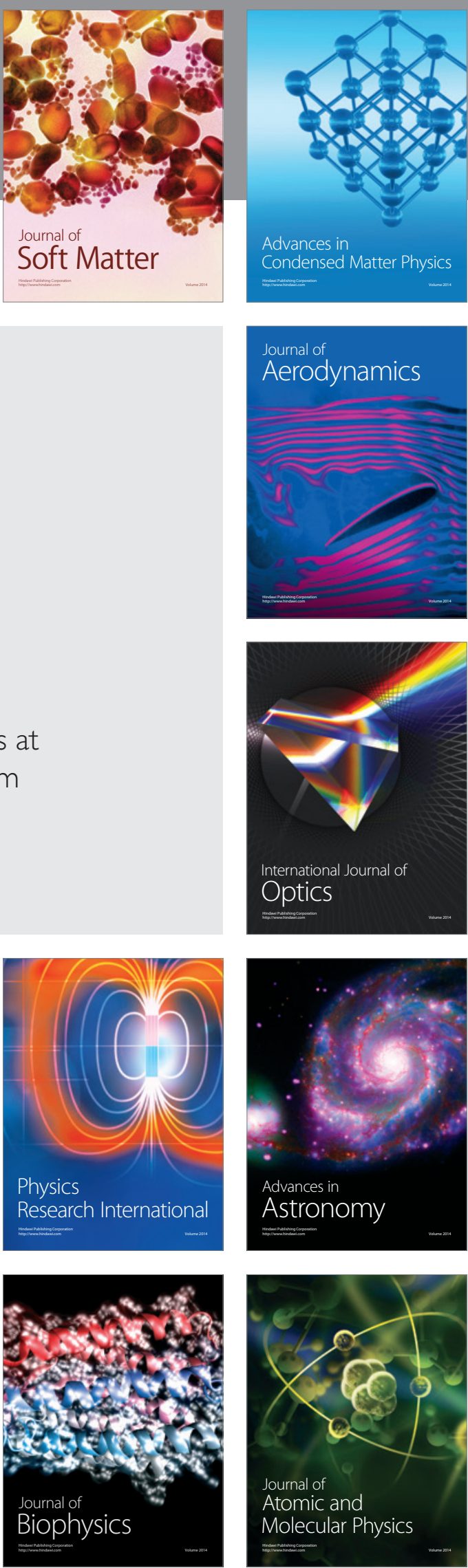